\title{
NEW ANALYTICAL RESULTS FOR THE ELECTROMAGNETIC RESPONSE OF A COMPOSITE SUPERCONDUCTING WIRE IN PARALLEL FIELDS
}

\author{
E.M.J. Niessen and P.J. Zandbergen \\ University of Twente, Dept. of Applied Mathematics \\ P.O. Box 217, 7500 AE Enschede, The Netherlands
}

\begin{abstract}
New analytical results are presented concerning the electromagnetic response of a composite superconducting wire in fields parallel to the wire axis. The basic elements of a description are the Maxwell equations supplemented with constitutive equations. The problem is non-linear due to the non-linearity in the constitutive equation describing the superconducting filaments. We show that by analytical means the non-linear behaviour of the wire can be studied. The new method gives an exact description of the response of a wire with non-conducting matrix material surrounding filaments with zero filament radius. For a composite wire it provides a very good approximation.
\end{abstract}

\section{INTRODUCTION}

In [1] a general model and calculation technique are presented for calculating the electromagnetic response of composite superconducting wires. The geometry considered is an infinitely long, circular, composite superconducting wire with its axis parallel with the cylindrical $z$-axis. We also consider this geometry and the presented model.

The wire (radius $R$ ) consists of many filaments of superconducting material (radius $R_{f}$ ) embedded in normal conducting matrix material. The critical state model [2] describes the non-linear constitutive relation of a filament. In composite superconducting wires, Carr descibes the electromagnetic response by averaged quantities where the averaging takes place over an area containing a filament and the surrounding part of the matrix [3]. Input for the model is a constitutive equation for a single superconducting filament, which can be found applying the critical state model. Carr's model will be used in this paper.

Wires subjected to parallel applied a.c. fields have not been studied very comprehensively $[4,5]$. Recent references, containing mainly numerical results, show nonexpected effects $[1,6]$. In [1] a first comparison between numerical and analytical data is given. However, the applied analytical method is linear. The new analytical method includes the non-linear conductivity properties.

We first present the set of equations describing the problem. Then we explain the basic idea's behind the new analytical method and apply the method to several situations. For a comprehensive treatment, see [7].

\section{SET OF EQUATIONS}

We introduce the cylindrical coordinate system $(r, \phi, z)$ with the $z$-coordinate parallel to the wire axis. Because

Manuscript received August 24, 1992 the wire is twisted with twist length $L_{p}$, we define a second local coordinate system with one unit vector, $\vec{e}_{\|}$, parallel to the filament direction. With $\psi$ representing the local twist angle, i.e. $\tan \psi=\beta r$, with $\beta \equiv 2 \pi / L_{p}$, the transformation between both systems reads:

$$
\left(\vec{e}_{r}, \vec{e}_{\perp}, \vec{e}_{\|}\right)=\left(\vec{e}_{r}, \cos \psi \vec{e}_{\phi}-\sin \psi \vec{e}_{z}, \sin \psi \vec{e}_{\phi}+\cos \psi \vec{e}_{z}\right)
$$

The normal conducting matrix material surrounding the filaments has an isotropic linear electrical conductivity $\sigma$. The non-linear constitutive equation describing the superconducting filaments is given by:

$$
j_{\|}^{s}= \begin{cases}j_{c} E_{\|} / E_{1} & \text { if }\left|E_{\|}\right| \leq E_{1} \\ j_{c} \operatorname{sign}\left(E_{\|}\right) & \text {if }\left|E_{\|}\right|>E_{1}\end{cases}
$$

with $E_{1}=R_{f}\left|\partial_{t} B_{\|}\right| / 4$ for parallel applied magnetic fields [7]. Here $j_{\|}^{s}$ and $E_{\|}$are the superconducting current density and the electric field parallel to the filament. If $\left|E_{\|}\right| \leq E_{1}$ the filament is denoted unsaturated, else it is denoted saturated. The constitutive equations for the composite finaly read: $j_{r}=\sigma_{\perp} E_{r}$ and

$$
\left(j_{\phi}, j_{z}\right)=\left(\beta r j_{s}+\sigma_{\phi \phi} E_{\phi}+\sigma_{\phi z} E_{z}, j_{s}+\sigma_{\phi z} E_{\phi}+\sigma_{z z} E_{z}\right)
$$

with $j_{s}=\eta j_{\|}^{s} \cos \psi, \sigma_{\perp}=(1-\eta) /(1+\eta) \sigma, \sigma_{\|}=(1-$ $\eta) \sigma, \sigma_{\phi \phi}=\sigma_{\perp}+\Delta \sigma \sin ^{2} \psi, \sigma_{\phi z}=\Delta \sigma \sin \psi \cos \psi, \sigma_{z z}=$ $\sigma_{\|}-\Delta \sigma \sin ^{2} \psi$, and $\Delta \sigma=\sigma_{\|}-\sigma_{\perp}$. Here $\eta$ is the volume fraction of superconducting material. We consider a thin insulating layer present between filament and matrix.

In the rest of the paper we consider zero filament radius, except where especially denoted. Then $E_{\|}$is zero in the unsaturated region, resulting in: $E_{z}=-\beta r E_{\phi}$. The current density then follows from:

$$
\left(j_{r}, j_{\phi}, j_{z}\right)=\left(\sigma_{\perp} E_{r}, \beta r j_{s}+\sigma_{\perp} E_{\phi}, j_{s}+\sigma_{\perp} E_{z}\right)
$$

The applied magnetic field is chosen to be rotational symmetric and uniform in space (i.e. $\phi$ - $z$ invariant): $\left(B_{r}^{A}, B_{\phi}^{A}, B_{z}^{A}\right)=(0,0, f(t))$. Two cases are considered with respect to the time dependence: $f(t)=\alpha t$, with $\alpha$ a constant, or $f(t)=B_{0} \sin \omega t$, i.e. $B_{z}^{A}$ changes linearly in time or is harmonic. The $\phi-z$ invariant Maxwell equations read:

$$
\begin{aligned}
& \partial_{r} E_{z}=\partial_{t} B_{\phi} \quad\left(\partial_{r} r E_{\phi}\right) / r=-\partial_{t} B_{z} \\
& -\partial_{r} B_{z}=\mu_{0} j_{\phi} \quad\left(\partial_{r} r B_{\phi}\right) / r=\mu_{0} j_{z}
\end{aligned}
$$

Here $\vec{B}$ consists of an applied field $\vec{B}^{A}$ and induced field $\vec{B}^{I}$. We immediately get $E_{r}=B_{r}=0$ irrespective of the time dependence of $B_{z}^{A}[6]$. The boundary conditions read:

$$
\begin{aligned}
E_{\phi}(r=0, t) & =B_{\phi}(r=0, t)=0 \\
2 \pi R B_{\phi}(R, t) & =\mu_{0} I^{A}(t), \\
B_{z}(R, t) & =B_{z}^{A}(R, t)=f(t)
\end{aligned}
$$


Eq.(3) defines the applied current $I^{A}(t)$. Eq.(4) follows from rotational symmetry [7].

The calculations are performed using the standard parameter setting: $R=10^{-3} \mathrm{~m}, L_{p}=10^{-1} \mathrm{~m}, \eta=0.5$, $\sigma=10^{9} \Omega^{-1} m^{-1}, j_{c}=10^{9} A m^{-2}, \alpha=1 T s^{-1}, B_{0}=4 T$, $\omega=1 s^{-1}$ and $I^{A}=0$.

\section{BASIS OF THE NEW ANALYTICAL METHOD}

For the given setting we can neglect the normal currents compared to the superconducting currents. This means that we only consider the twisted superconducting filaments and no transient effects (following from normal currents) are present [7]. Then the total non-linear problem consists of two piecewise linear problems: 1) the unsaturated and 2) the saturated regime.

The general form of the solutions in both regimes can be found independently. The only (numerical) input is the number and general position(s) of the interface(s). Using the boundary conditions and matching the solutions in separate regions, gives the total solution, containing the position(s) of the interface(s) between the different regions.

In earlier literature the non-linear effects are removed introducing a linear surface current: saturation is neglected and the total wire is considered unsaturated. This approximation is only valid for very small saturated regions.

We consider the applied field linearly increasing in time and harmonic in time. Also an applied current will be considered. The basic idea, however, remains the same.

$$
\text { IV: } B_{z}^{A}=\alpha t, I^{A}=0
$$

$B_{z}^{A}$ increases linear in time: $f(t)=\alpha t$, with $\alpha>$ 0 . The initial conditions correspond to the virgin state: $\vec{E}(t \leq 0)=\vec{B}(t \leq 0)=\overrightarrow{0}$. The solutions for increasing and decreasing applied field are anti-symmetrical, because $I^{A}=0$.

We first consider the physics of the problem. At time $t=0$ the wire is completely unsaturated. As $t$ increases a negatively saturated region, that starts at the wire surface, grows inwards, while the interior remains unsaturated, see Figure 1. The position of the interface between the unsaturated and the negatively saturated region is denoted by $R_{1}(t)$. When the applied field reaches the level: $B_{z}^{A}=\mu_{0} \eta j_{c} /(2 \beta)[6]$, also a positively saturated region (interface $R_{2}(t)$ ) grows from the center of the wire outwards. This saturation in the inner region occurs after a change in $B_{z}^{A}$ independent of $\partial_{t} B_{z}^{A}[6]$.

We start with one interface $R_{1}$. The unsaturated region is given by $0 \leq r \leq R_{1}$. The unsaturation condition implies: $\partial_{t} B_{\phi}=\beta r \partial_{t} B_{z}$. Starting from the virgin state we obtain: $B_{\phi}=\beta r B_{z}$, so $B_{\perp}$ is zero. With $g(x) \equiv \sqrt{1+\beta^{2} x^{2}}$, we obtain: $E_{\phi}=-\alpha \ln (g(r)) /\left(\beta^{2} r\right)$ and $B_{z}=\alpha t / g^{2}(r)$.

In the negatively saturated region $R_{1}<r \leq R$, we find

$$
\begin{aligned}
& E_{\phi}=-\alpha\left(r^{2}-R_{1}^{2}\right) /(2 r)-\alpha \ln \left(g\left(R_{1}\right)\right) /\left(\beta^{2} r\right) \\
& E_{z}=E_{z}\left(R_{1}\right)
\end{aligned}
$$

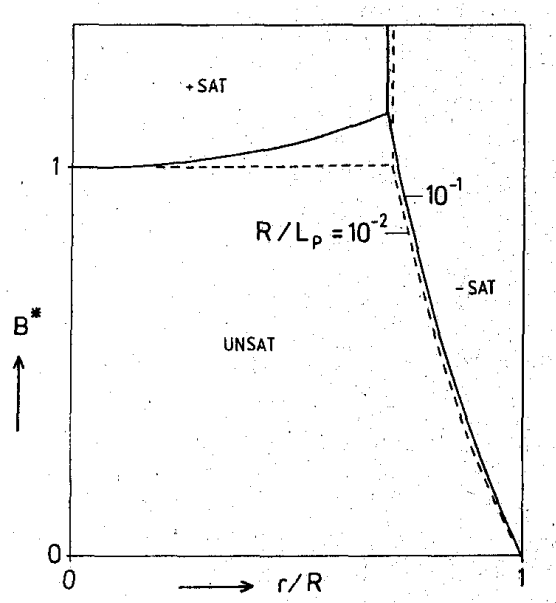

Figure 1: The positions of the interfaces for $B_{z}^{A}=\alpha t$, $\alpha>0$, as function of the normalized magnetic field $\left(B^{*}=\right.$ $\left.4 \pi \alpha t /\left(\mu_{0} \eta j_{c} L_{p}\right)\right)$

$$
\begin{aligned}
& B_{z}=\alpha t-\mu_{0} \eta j_{c}[g(R)-g(r)] / \beta \\
& B_{\phi}=\mu_{0} \eta j_{c}[g(R)-g(r)] /\left(\beta^{2} r\right)
\end{aligned}
$$

$R_{1}(t)$ follows from continuity of $B_{z}$ at $R_{1}$ :

$$
\mu_{0} \eta j_{c} g^{2}\left(R_{1}\right)\left[g(R)-g\left(R_{1}\right)\right]=\alpha t \beta^{3} R_{1}^{2}
$$

$R_{2}(t)$ follows from conservation of current:

$\eta j_{c} \mu_{0}\left[g\left(R_{2}\right)-1-g(R)+g\left(R_{1}\right)\right]=\frac{\alpha t \beta}{g^{2}\left(R_{1}\right)}-\frac{\alpha t \beta}{g^{2}\left(R_{2}\right)}(6)$

Notice that $R_{1}$ can be found independently from $R_{2}$, eq.(5), which is not the case with $R_{2}$, eq.(6).

$$
\text { V. } B_{z}^{A}=B_{0} \sin \omega t, I^{A}=0
$$

Now $f(t)$ is harmonic: $f(t)=B_{0} \sin \omega t$. Numerical calculations show that the response of the wire is also a periodic function of time (see Figure 2). The figure also indicates that when $\partial_{t} B_{z}^{A}$ changes sign the saturated region does not shrink from the interior towards the wire surface, but a second, oppositely saturated region grows from the wire surface inwards. The first saturated region moves inwards and diminishes.

We describe an elementary phase interval $w t \in$ $(\pi / 2,3 \pi / 2)$. From the center of the wire outwards, the wire is unsaturated $\left(0 \leq r \leq R_{1}\right)$, negatively saturated $\left(R_{1}<r<R_{3}\right)$ and positively saturated $\left(R_{3}<r \leq R\right)$. Considering the standard setting the amplitude $B_{0}$ is small enough not to saturate the center of the wire.

The analyses of the unsaturated and the positively sat urated region follow straightforwardly from the previous section. With $k \equiv\left(\mu_{0} \eta j_{c} R_{3} / g\left(R_{3}\right)\right) d R_{3} / d t$, the expressions in the negatively saturated region read [7]

$E_{\phi}=R_{1} E_{\phi}\left(R_{1}\right) / r+\left(r^{2}-R_{1}^{2}\right)\left[2 \beta k-B_{0} \omega \cos \omega t\right] /(2 r)$ 


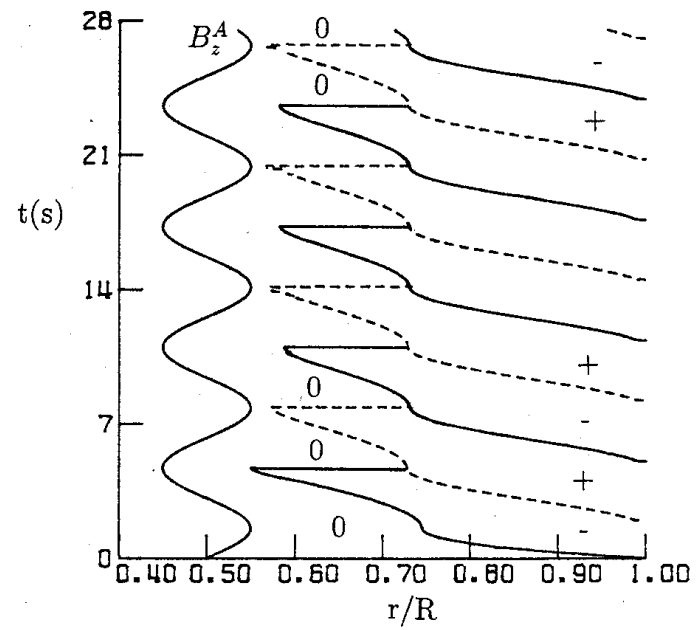

Figure 2: Position of the interfaces for $B_{z}^{A}=B_{0} \sin \omega t[6]$.

$$
\begin{aligned}
& E_{z}=E_{z}\left(R_{1}\right)+2 k \ln \left(r / R_{1}\right) \\
& B_{\phi}=R_{3} B_{\phi}\left(R_{3}\right) / r+\mu_{0} \eta j_{c}\left[g\left(R_{3}\right)-g(r)\right] /\left(\beta^{2} r\right) \\
& B_{z}=B_{z}\left(R_{3}\right)-\mu_{0} \eta j_{c}\left[g\left(R_{3}\right)-g(r)\right] / \beta
\end{aligned}
$$

Utilizing $B_{\phi}\left(R_{1}\right)=\beta R_{1} B_{z}\left(R_{1}\right)$ we obtain:

$$
\mu_{0} \eta j_{c} g^{2}\left(R_{1}\right)\left[-g(R)+2 g\left(R_{3}\right)-g\left(R_{1}\right)\right]=\alpha t \beta^{3} R_{1}^{2}
$$

Observe that for $R_{3}=R$ this equaton is identical to eq.(5). $E_{\|}$is zero on the interface $R_{3}$ resulting in:

$$
\left[2 \beta^{2} k-\beta B_{0} \omega \cos \omega t\right]\left(R_{3}^{2}-R_{1}^{2}\right)+4 k \ln \left(R_{3} / R_{1}\right)=0
$$

with initial condition: $R_{3}(\omega t=\pi / 2)=R$. Using eq.(7) and (8), $R_{1}, R_{3}$ and $d R_{3} / d t$ can be found.

Notice that $R_{3}$ is a function of time because of the time dependence of the applied field. The twist is responsible for the time dependence of $R_{1}$.

$$
\text { VI. } B_{z}^{A}=\alpha t, I^{A}<0
$$

Starting with a virgin wire and no applied magnetic field, the current in the wire is decreased. When $I^{A}$ does not decrease too much, the center remains unsaturated while a negatively saturated region (interface $R_{1}$ ) grows from the boundary $R$ inwards. The symmetry between the solutions for increasing and decreasing applied magnetic field is broken due to the non-zero applied current.

Case: $\alpha>0$. Increasing the magnetic field results in a further growth of the negatively saturated region $\left(R_{1}<r \leq R\right) . \quad R_{1}(t)$ is given by eq.(5) with a modified right hand side: $\alpha t \beta^{3} R_{1}^{2}-\beta^{2} \mu_{0} I^{A} /(2 \pi)$. The effect of an applied current can not simply be introduced as an effective applied magnetic field because in the last equation $B_{z}^{A}=\alpha t$ is coupled with $R_{1}$ while $I^{A}$ is not.

Case: $\alpha<0$. Decreasing the magnetic field results in the appearance of three regions: unsaturated $\left(0 \leq r \leq R_{1}\right)$, negatively saturated $\left(R_{1}<r<R_{3}\right)$ and positively saturated $\left(R_{3}<r \leq R\right)$, similar to the previous section. We obtain eq.(7) with a modified right hand side: $\alpha t \beta^{3} R_{1}^{2}-\beta^{2} \mu_{0} I^{A} /(2 \pi)$, while the second relation is still given by eq.(8), with $R_{3}(t=0)=R$.

\section{RESULTS AND CONCLUSIONS}

We first compare numerical and analytical results. For

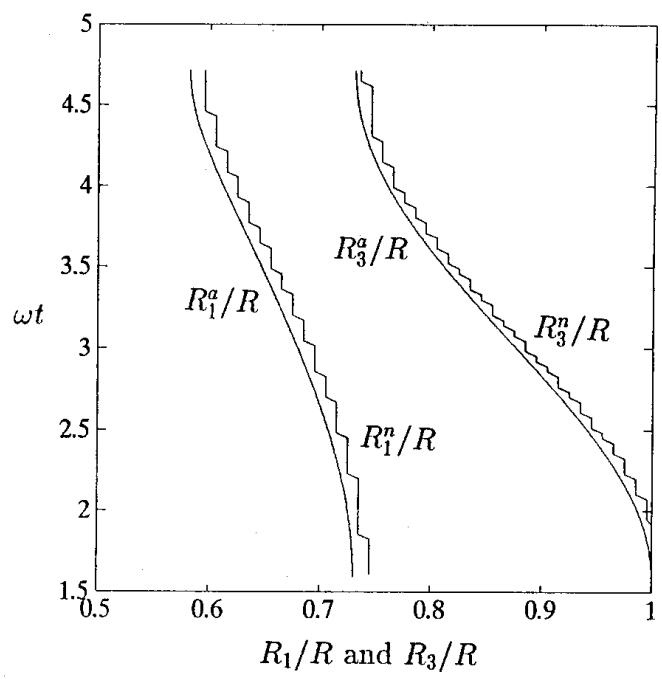

Figure 3: Positions of the interfaces $R_{1}$ and $R_{3}$ for $B_{z}^{A}=$ $B_{0} \sin \omega t$; numerical (n) and analytical (a) data.

the harmonic applied magnetic field the interfaces $R_{1}$ and $R_{3}$ are given in Figure 3. The 'jumps' in the numerically obtained lines follow from discretization errors. There is very good agreement between both results. The maximum and minimum value of $R_{1}$ are strong non-linear functions of $B_{0}$, as shown in Figure 4. We consider the dissipation

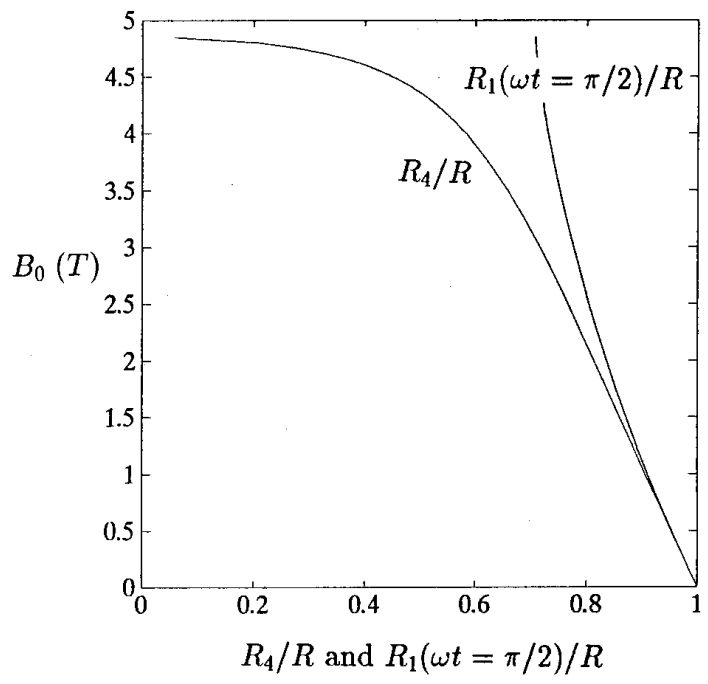

Figure 4: Maximum $\left(R_{1}(\omega t=\pi / 2)\right)$ and minimum value $\left(R_{4}=R_{1}(\omega t=3 \pi / 2)\right)$ of $R_{1} . B_{z}^{A}=B_{0} \sin \omega t$. 
in the wire placed in a harmonic applied magnetic field. The mean loss per unit length is given by:

$$
\bar{P}=\frac{\omega}{2 \pi} \int_{0}^{2 \pi / \omega} \int_{0}^{R} \int_{0}^{2 \pi} \sigma_{\|} E_{\|}^{2}+\sigma_{\perp} E_{\perp}^{2}+\eta j_{\|}^{s} E_{\|} r d r d \phi d t
$$

In Figure 5 the scaled loss $2 \pi \bar{P} /\left(\omega^{2} B_{0}^{2}\right)$ is given as function of $B_{0}$. For the numerical data the grid sizes are:

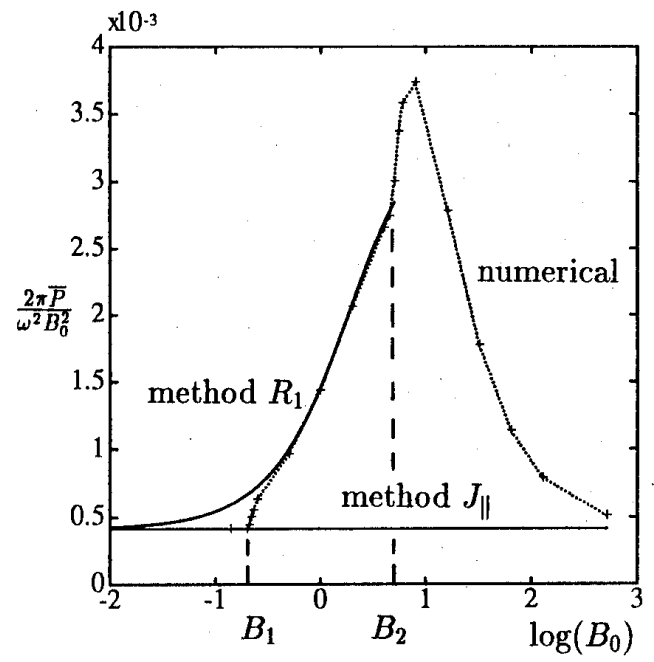

Figure 5: $2 \pi \bar{P} /\left(\omega^{2} B_{0}^{2}\right)$ as function of $\log \left(B_{0}\right)$.

$\Delta r=R / 50$ and $\Delta t=2 \pi / 200$. Also the results of two analytical calculations are shown in the figure. The constant horizontal line is calculated using the surface current approach $[1,6]$ (method $\left.J_{\|}\right)$. The other solid line follows from the new analytical method (method $R_{1}$ ). For small values of $B_{0}$ there is excellent agreement between all three results because the saturated region is negligibly small, so the problem is linear. On the numerical grid no saturation occurs. The only loss term that contributes is $\sigma_{\perp} E_{\perp}^{2}$. The deviation between the numerical and $J_{\|}$ method starts at $B_{0}=B_{1}$ because for $B_{0}>B_{1}$ outer grid cells saturate. The loss terms $\sigma_{\|} E_{\|}^{2}$ and $\eta j_{\|}^{s} E_{\|}$then also contribute. Method $J_{\|}$however, does not take these extra losses into account because the surface current is considered to be unsaturated. Method $R_{1}$ already deviates for smaller $B_{0}$ values because it does not have the threshold behaviour of first appearence of non-linear behaviour when the first outer grid cell saturates.

For increasing amplitude $B_{0}$, the saturated regions penetrate more towards the center of the wire. For $B_{0}=B_{2}$ also a saturated region grows outwards from the center of the wire and the losses increase even more.

For very large values of $B_{0}$ the term $E_{\|} j_{\|}^{s}$ can be neglected compared to the Ohmic loss terms. The linear behaviour is dominant and the deviation between numerical and analytical $J_{\|}$results decreases.

For a linear increasing applied magnetic field, $R_{1}(t)$ is calculated for several values of the filament radius. As constitutive equation, we used eq.(1). Figure 6 indicates that the saturation effects do not change drastically for non-zero filament radius and that they are worse for zero filament radius.

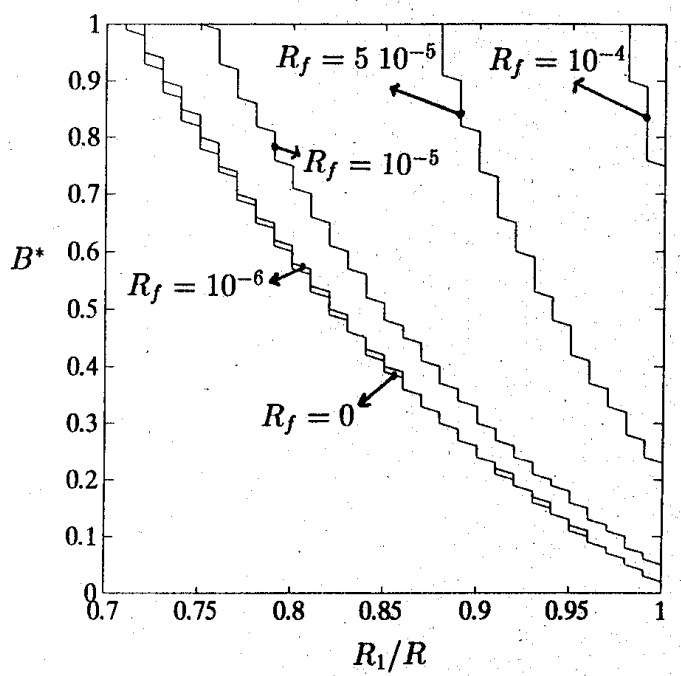

Figure 6: $R_{1}$ as function of $B^{*}\left(=4 \pi \alpha t /\left(\mu_{0} \eta j_{c} L_{p}\right)\right)$ for several values of the filament radius $R_{f}$.

It can be concluded that the new method gives a very accurate description of the non-linear response of the wire.

\section{REFERENCES}

[1 ] L.J.M. van de Klundert, E.M.J. Niessen and P.J. Zandbergen, "Electromagnetic response of composite superconducting wires," Journal of Engineering Mathematics 26, pp. 231-265, 1992.

[2 ] C.P. Bean, "Magnetization of hard superconductors," Phys. Rev. Lett., vol. 8, pp. 250-253, 1962.

[3 ] W.J. Carr, jr., "Electromagnetic theory for filamentary superconductors," Phys. Rev. B 11, pp. 1547$1554,1975$.

[4 ] B. Turck, "Courants de circulation et pertes dans les composites supraconducteurs soumis a une induction longitudinale variable," Revue de physiquee appliquée, pp. 369-375, May 1976.

[5 ] G. Ries and K.P. Jüngst, "Filament coupling in multifilamentary superconductors in pulsed longitudinal fields," Cryogenics 26 (1976).

[6 ] L.J.M. van de Klundert, "New developments on calculating and measuring AC losses in composite superconductors," IEEE transactions on magnetics, vol. 28 , no. 1,1992 , pp. 705-710.

[7 ] E.M.J. Niessen and P.J. Zandbergen, "Analytical results for the electromagnetic response of a composite superconducting wire in parallel fields," Memorandum No. 1059, University of Twente, The Netherlands, (1992) ISSN 0169-2690. 